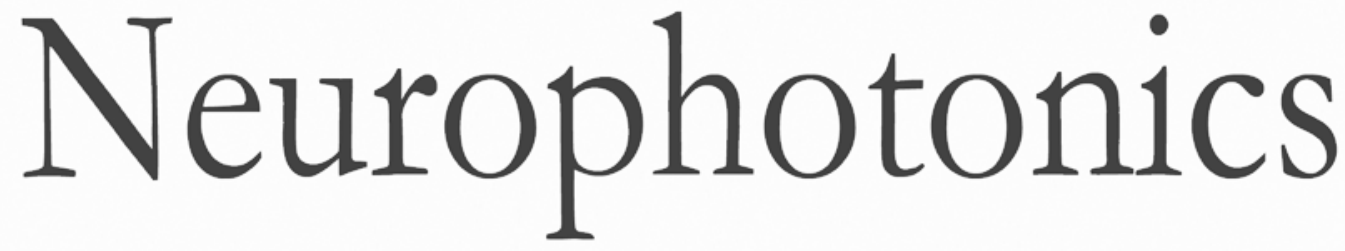

\title{
Longitudinal detection of retinal alterations by visible and near- infrared optical coherence tomography in a dexamethasone- induced ocular hypertension mouse model
}

Weiye Song

Sipei Fu

Shangshang Song

Sui Zhang

Lei Zhang

Steven Ness

Manishi Desai

Ji Yi 


\title{
Longitudinal detection of retinal alterations by visible and near-infrared optical coherence tomography in a dexamethasone-induced ocular hypertension mouse model
}

\author{
Weiye Song, ${ }^{a}$ Sipei Fu, ${ }^{b}$ Shangshang Song, ${ }^{\mathrm{c}}$ Sui Zhang, ${ }^{\mathrm{d}}$ Lei Zhang, ${ }^{\mathrm{a}}$ Steven Ness, ${ }^{\mathrm{e}}$ Manishi Desai, ${ }^{\mathrm{e}}$ and Ji Yja,f,* \\ ${ }^{a}$ Boston University School of Medicine, Boston Medical Center, Department of Medicine, Boston, Massachusetts, United States \\ ${ }^{\mathrm{b} B o s t o n}$ University, Department of Biology, Boston, Massachusetts, United States \\ ${ }^{\mathrm{c} B o s t o n}$ University Sargent School of Rehabilitation, Department of Health Science, Boston, Massachusetts, United States \\ ${ }^{\mathrm{d} D a n a-F a r b e r}$ Cancer Institute, Boston, Massachusetts, United States \\ 'Boston Medical Center, Department of Ophthalmology, Boston, Massachusetts, United States \\ 'Boston University, Department of Biomedical Engineering, Boston, Massachusetts, United States
}

\begin{abstract}
The retina, as part of the central nervous system, has distinct anatomical and structural properties for its visual function. Light scattering spectroscopy, while widely used for tissue structural characterization and disease diagnosis, has been relatively unexplored in the living retina. Recently, we have developed a fiber-based visible and near-infrared optical coherence tomography system (vnOCT) for in vivo retinal imaging, to uniquely measure a spectroscopic marker (VN ratio) sensitive to nanoscale pathological changes. In the present study, we applied vnOCT in an animal model of glaucoma (dexamethasone-induced ocular hypertension mouse) and tested the capabilities of four optical markers, VN ratio, peripapillary retinal nerve fiber layer (RNFL) thickness, total retinal blood flow, and hemoglobin oxygen saturation $\left(\mathrm{sO}_{2}\right)$, for the detection of retinal ganglion cell (RGC) damage in association with ocular hypertension. We found that RNFL-RGC VN ratio and arteriovenous (A-V) $\mathrm{sO}_{2}$ are capable of detecting early retinal alteration in ocular hypertensive eyes, preceding measurable change of RNFL thickness. This study suggests a potential clinical application of vnOCT in early detection of glaucoma. (C) The Authors. Published by SPIE under a Creative Commons Attribution 4.0 Unported License. Distribution or reproduction of this work in whole or in part requires full attribution of the original publication, including its DOI. [DOI: 10.1117/1.NPh.6.4.041103]
\end{abstract}

Keywords: visible and near-infrared optical coherence tomography system; hemoglobin oxygen saturation; VN ratio; ocular hypertension.

Paper 19006SSRR received Jan. 25, 2019; accepted for publication Jun. 12, 2019; published online Jul. 9, 2019.

\section{Introduction}

Glaucoma is an irreversible progressive optic neuropathy associated with loss of retinal ganglion cells (RGCs). ${ }^{1}$ It is known as "the silent thief of sight," since there are usually no symptoms until vision impairment has occurred and becomes irreversible. Typically by the time functional changes are detected, $30 \%$ of RGCs have already been lost. ${ }^{2,3}$ As early treatment can significantly reduce the risk of glaucoma, preclinical detection prior to the onset of functional visual field defects holds the key for prevention of vision loss. ${ }^{4,5}$

Optical coherence tomography (OCT) has become a standard-of-care test for glaucoma management and plays an important role in disease diagnosis by identifying thinning in the peripapillary retinal nerve fiber layer (RNFL) ${ }^{6-8}$ While OCT imaging is capable of detecting thickness change prior to development of measurable peripheral field loss, RNFL thinning alone is not sensitive enough to differentiate early glaucoma from the high-risk glaucoma suspect who may benefit from earlier treatment. ${ }^{9}$ Recent advances in optical coherence tomography angiography (OCTA) have directed attention to measuring capillary flow index and capillary density in the peripapillary region, ${ }^{10-15}$ but the sensitivity of these markers for early detection of glaucoma is still under investigation. Meanwhile, the

*Address all correspondence to Ji Yi, E-mail: jiyi@bu.edu development of visible light OCT (vis-OCT) has drawn interest in the ophthalmic applications for several distinct advantages recently. ${ }^{16-18}$ First, due to the distinct absorption spectra of oxyand deoxyhemoglobin in the visible light range, this technology allows functional measurement of both hemoglobin oxygen saturation $\left(\mathrm{sO}_{2}\right)$ and (in combination with blood flow measurements) the inner retinal oxygen metabolic rate $\left(\mathrm{MRO}_{2}\right) .{ }^{19}$ Second, by comparing the reflected signals between visible light and near-infrared (NIR) OCT images, the light scattering spectroscopic (LSS) contrast can detect ultrastructural changes in the retina beyond the resolution limit of current commercial imaging devices. ${ }^{20-22}$ This LSS contrast can be quantified by a novel optical marker, called the $\mathrm{VN}$ ratio, that normalizes the visible light OCT images with NIR ones. Previous data suggest that pathologic changes in the RGC cytoskeleton occur at a length scale $<300 \mathrm{~nm}$, and the use of LSS contrast or VN ratio can allow us to optically detect structural alteration prior to RNFL thinning. ${ }^{23,24}$ Lastly, vis-OCT can achieve 1 to $2 \mu$ m axial resolution, ${ }^{17,25}$ offering the potential to detect subtle RNFL thickness changes and refine current measurement capabilities.

To fully utilize the advantages of vis-OCT, we have developed and implemented a fiber-based visible and near-infrared OCT (vnOCT) for simultaneous dual-band retinal imaging. ${ }^{21}$ The combination of the two bands allows multimetric measurement of the abovementioned spectroscopic markers, including $\mathrm{VN}$ ratio in the RNFL-RGC complex and $\mathrm{sO}_{2}$ in the inner retinal circulation. The purpose of this study is to apply vnOCT in 
a dexamethasone-induced ocular hypertension mouse model of early glaucoma and to explore whether the spectroscopic markers can detect changes of retinal ultrastructure or circulation. We compared intraocular pressure (IOP) and RGC loss in dexamethasone-treated and control mice and longitudinally measured $\mathrm{sO}_{2}$, retinal blood flow, RNFL thickness, and $\mathrm{VN}$ ratio. The alterations and relation of these metrics may provide valuable insight into the pressure-related pathophysiology of glaucoma and lead to the development of optical markers that can translate into early clinical detection of glaucoma.

\section{Methods}

All animal procedures were approved by the Institutional Animal Care and Use Committee at Boston Medical Center and conformed to the guidelines on the use of animals from the NIH and the ARVO Statement for the Use of Animals in Ophthalmic and Vision Research.

\subsection{Visible and Near-Infrared Optical Coherence Tomography Setup}

The dual-band OCT setup used in this study is modified from our previous publication, ${ }^{21}$ as shown in Fig. 1. Briefly, a supercontinuum laser provided both visible light and NIR illumination, from 540 to $600 \mathrm{~nm}$ and 800 to $875 \mathrm{~nm}$, respectively. The visible light was separated from the NIR by a dichroic mirror (DMLP650R, Thorlabs), dispersed by a pair of prisms, and filtered by a slit aperture. The filtered light was reflected by a mirror and coupled in an optical fiber. The NIR range was also separated by another dichroic mirror (DMLP900R, Thorlabs), filtered by two edge filters (DMLP805, Thorlabs and 19354, Edmund Optics), and coupled into an optical fiber. The two

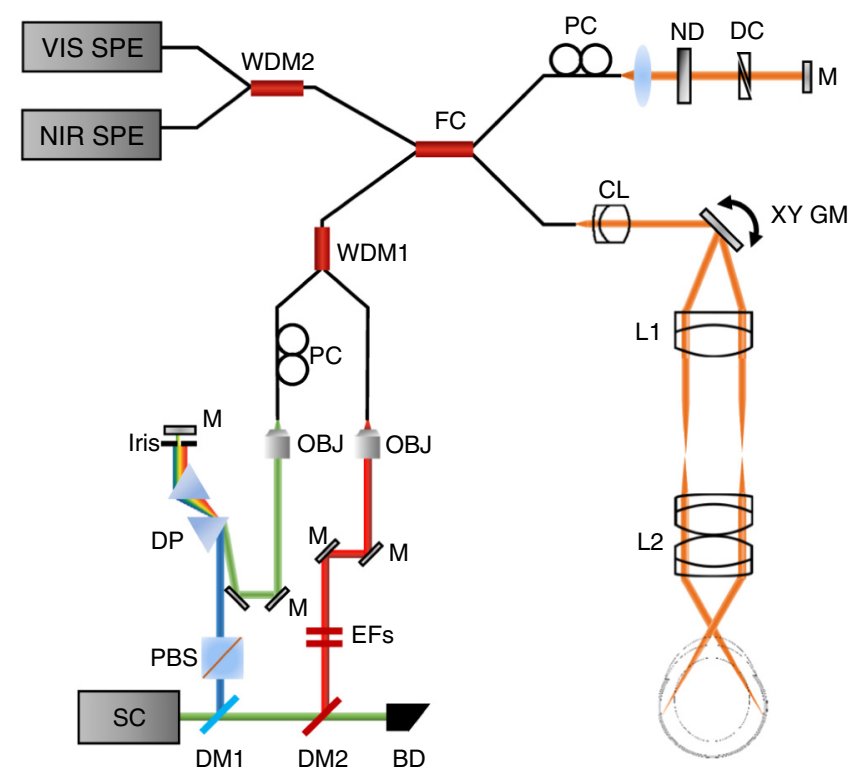

Fig. 1 Schematic of the vnOCT system setup. Two WDM and a fiber coupler merge and split the visible and NIR channels. The same sample and reference arms are shared by two channels, which are collected by separate spectrometers. SC, supercontinuum source; $\mathrm{DM}$, dichroic mirror; BD, beam dumper; PBS, polarization beam splitter; DP, dispersive prism; M, mirror; EFs, two edge filters; OBJ, objective lens; PC, polarization controller; CL, collimating lens; GM, galvanometer mirror; L, lens; ND, neutral density filter; DC, dispersion compensation plates; VIS SPE, visible band spectrometer; NIR SPE, near-infrared band spectrometer. bands were combined by a custom-made wavelength division multiplexer (WDM) and directed into a 90/10 optical fiber coupler (TW670R2A2, Thorlabs). The returning light was separated by another WDM for the visible and NIR channels and recorded by two separate spectrometers equipped with 2048 pixel line scan cameras (Basler, splk2048-140k) and running at a $50-\mathrm{kHz}$ A-line rate. Because both bands propagate within the same optical fiber, they shared the same sample arm and reference arm. The sample arm consisted of a collimator $(f=6 \mathrm{~mm})$, a two axis galvanometer scanner (GVSM002, Thorlabs), and a $3: 1$ telescope $(f=75 \mathrm{~mm}$ and $f=25 \mathrm{~mm})$ to relay light to the pupil. The reference arm consisted of a collimator $(f=10 \mathrm{~mm})$, several dispersion control BK-7 plates, a variable neutral density filter, and a mirror to reflect the light. The beam size on the pupil is about $0.2 \mathrm{~mm}$ in diameter. The incident optical power on the mouse pupil is 0.3 and $0.8 \mathrm{~mW}$, in the visible light and NIR bands, respectively. The exposure time of the camera was $17 \mu$ s for an A-line rate of $50 \mathrm{kHz}$. The field of view was $1.2 \times 1.2 \mathrm{~mm}^{2}$. The axial and lateral resolutions were estimated to be 2.7 and $6.7 \mu \mathrm{m}$ for the visible band, 5.6 and $10.2 \mu \mathrm{m}$ for NIR band, respectively. The system acquires visible and NIR OCT images simultaneously.

\subsection{In Vivo Retinal Imaging}

For imaging, we anesthetized mice with a mixture of ketamine and xylazine (ketamine: $11.45 \mathrm{mg} \mathrm{mL}^{-1}$; xylazine: $1.7 \mathrm{mg} \mathrm{mL}^{-1}$, in saline) that was injected intraperitoneally at a dose of $10 \mathrm{~mL} /$ $\mathrm{kg}$ body weight. After anesthesia, the animal was placed on a custom-made animal holder for imaging. We applied 1\% tropicamide hydrochloride ophthalmic solution to dilate the pupil. Commercial artificial tears were applied to the mice's eyes every other minute to prevent corneal dehydration. Imaging included two scanning protocols consecutively performed, including three-dimensional (3-D) OCT images in a $256 \times 256$ raster scanning pattern centered at the optic disk followed by a dual-circle scanning of two concentric circles repeated eight times with 4096 pixels in each circle. The optic nerve head (ONH) was aligned to be at the center of the field-of-view (FOV) so that all the major vessels could be imaged. Figures 1(a) and 1(b) exemplify the en face projection of visible and NIR OCT images acquired simultaneously. The 3-D OCT images were used for calculating $\mathrm{SO}_{2}$ and $\mathrm{VN}$ ratio. The dual-circle scanning data were used to calculate retinal blood flow and RNFL thickness. After imaging, the mice were released from the animal holder for recovery.

\subsection{Blood Hemoglobin Saturation $\left(\mathrm{sO}_{2}\right)$}

We used the previously published method for calculating $\mathrm{sO}_{2}$ from retinal arteries and veins, ${ }^{16,19}$ as shown in Fig. 2(c). The 3-D images from the visible light channel were used. A shorttime Fourier transform with a sweeping Gaussian window in $k$ spectral domain $(k=2 \pi / \lambda)$ was performed to generate wavelength-dependent vis-OCT images. The full width at half maximum of the $k$-domain Gaussian window was $k_{w}=0.32 \mu \mathrm{m}^{-1}$. We used 11 windows to generate four-dimensional data $(x, y, z, \lambda)$. The retinal surface was identified by setting an intensity threshold. To reduce the dimension, individual vessels were first segmented from the en face projection, and all the A-lines within each vessel were lined-up to a 2-D B-scan. Then each A-line was shifted in reference to the retinal surface. The signal from the bottom wall of each individual vessel was averaged, 
(a)

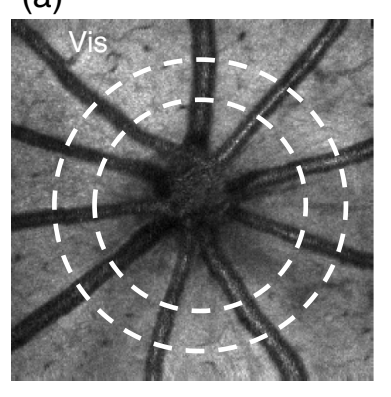

(b)

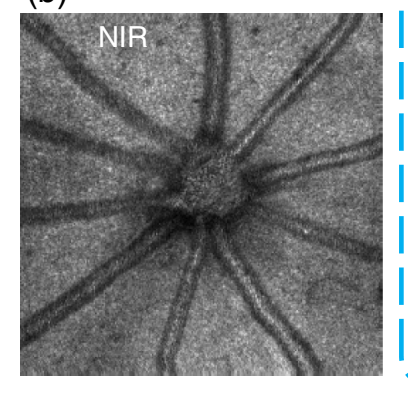

(c)

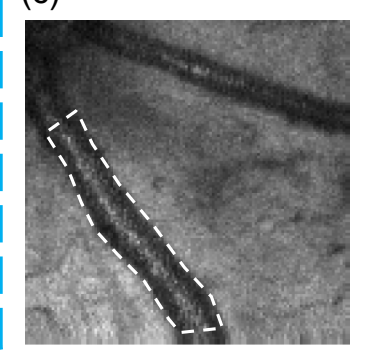

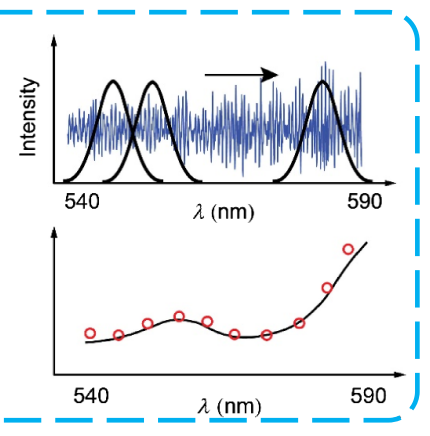

(e) 1
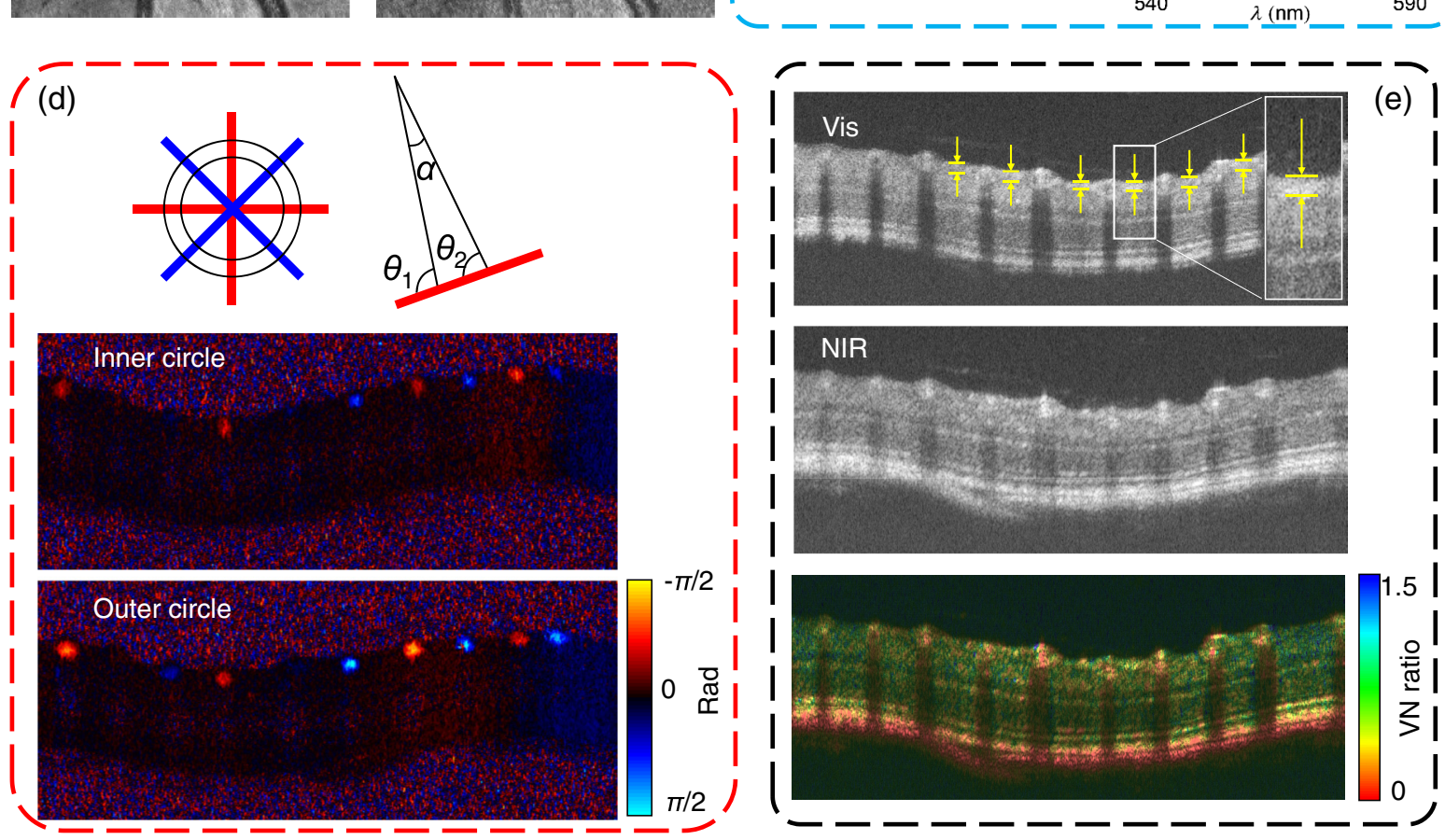

Fig. 2 Multimetric measurements by vnOCT. (a), (b) En face projection of mouse retina in the visible and NIR channels, respectively. Two concentric circles represent the dual-circle scanning pattern. (c) Method for $\mathrm{sO}_{2}$ calculation by vessel segmentation and subsequent spectroscopic analysis. The vessel location was first manually segmented, and short-time Fourier transform was performed to obtain 4-D data $(x, y, z, \lambda)$. Finally, the spectra from the bottom vessel wall were averaged and fitted by Eq. (1). (d) The schematic of blood flow measurements. The difference of the phase contrast from the inner and outer circles was used to calculate Doppler angle and blood velocity. (e) Illustration of RNFL thickness and RNFL-RRC VN ratio calculation. The circular B-scan image in visible light OCT was used to calculate RNFL thickness, as shown in yellow arrows. The intensity ratio between the visible and NIR channels was defined as $\mathrm{VN}$ ratio.

and the same process was iterated for each spectral window to generate a spectrum for each vessel. Lastly, the following analytical model was used to fit the spectrum to calculate $\mathrm{sO}_{2}$ from 545 to $580 \mathrm{~nm}$ :

$$
\begin{aligned}
I^{2}(\lambda)= & I_{0}^{2}(\lambda) R_{0} r(\lambda) \exp \left[-2 d \mu_{\mathrm{HbO}_{2}}(\lambda) \times \mathrm{sO}_{2}-2 d \mu_{\mathrm{Hb}}(\lambda)\right. \\
& \left.\times\left(1-\mathrm{sO}_{2}\right)\right]
\end{aligned}
$$

where $I_{0}(\lambda)$ is the incident spectrum on the retina; $R_{0}$ is the reference arm reflectance; $d(\mathrm{~mm})$ is the vessel diameter; and $r(\lambda)$ (dimensionless) is the reflectance at the vessel wall, whose scattering spectrum is modeled as a power law under the firstorder Born approximation $r(\lambda)=A \lambda^{-}$, where A (dimensionless) is a constant. ${ }^{26}$ The subscripts $\mathrm{Hb}$ and $\mathrm{HbO}_{2}$ denote the contribution from deoxygenated and oxygenated blood, respectively. The optical attenuation coefficient $\mu\left(\mathrm{mm}^{-1}\right)$ combines the absorption $\left(\mu_{\mathrm{a}}\right)$ and scattering coefficients $\left(\mu_{\mathrm{s}}\right)$ of the whole blood, which are both wavelength- and oxygenation-dependent: $\mu(\lambda)=\mu_{\mathrm{a}}(\lambda)+0.2 \mu_{\mathrm{s}}(\lambda)$.

The values of $\mu_{\mathrm{a}}$ and $\mu_{\mathrm{s}}$ are provided by the literature. ${ }^{27}$ A least-square linear regression to the logarithmic spectra returned the value of $\mathrm{sO}_{2}$. We averaged $\mathrm{sO}_{2}$ from all the arteries and veins, respectively, for each retina.

\subsection{Retinal Blood Flow}

We used the dual-circle scanning data from the NIR channel to calculate the blood flow, using the method of phase-sensitive Doppler OCT [Fig. 2(d)]. ${ }^{28-30}$ The scanning protocol scanned two concentric circles around the $\mathrm{ONH}$ at a radius of 0.21 and $0.32 \mathrm{~mm}$, respectively. ${ }^{31-33}$ At each circle, it first scanned clockwise and immediately counterclockwise, and the round trip was repeated four times. Each circular scan included 4096 A-lines. The phase delay between adjacent A-lines can measure the blood flow velocity, $v(\mathrm{~mm} / \mathrm{s})$ by 
$v=\frac{f_{\text {sample } \cdot \lambda_{\text {center }} \cdot \Delta \varphi}}{4 \pi n \cos \theta}$

where $f_{\text {sample }}$ is the OCT A-line scanning frequency, $\lambda_{\text {center }}(\mathrm{nm})$ is the center wavelength of the OCT light source at $820 \mathrm{~nm}, \Delta \varphi$ (rad) is the phase shift between adjacent A-scans, $n=1.38$ (dimensionless) is the refraction index of the sample, and the Doppler angle $\theta(\mathrm{rad})$ is the angle between the probing beam and the vessel.

The only unknown parameter in the right side of equation is the Doppler angle $\theta$. To estimate $\theta$, we used the phase difference between the two circles, ${ }^{34}$ as shown in Fig. 2(d). The blood flow velocity from the same vessel measured at the inner circle $v_{1}$, and the outer circle $v_{2}$, should be the same such that $v_{1}=v_{2}$. When plugging in Eq. (3), we have

$\frac{\Delta \varphi_{1}}{\Delta \varphi_{2}}=\frac{\cos \theta_{1}}{\cos \theta_{2}}$.

The difference between $\theta_{1}$ and $\theta_{2}$ is controlled by the galvanometer scanner that $\theta_{1}=\theta_{2}+\alpha$, where $\alpha=0.035$ (rad). Therefore, by taking the ratio of the phase difference between the two circles from the same vessel, $\theta_{1}$ can be calculated by

$\theta_{1}=\tan ^{-1}\left(\frac{\frac{\Delta \varphi_{1}}{\Delta \varphi_{2}}-\cos \alpha}{\sin \alpha}\right)$

The data processing follows several steps. First, the phase delays between adjacent A-lines were calculated after the Fourier transform. Second, the phase signals from the clockwise and counterclockwise circular scans were averaged after reverting the counterclockwise scan. The purpose of the round trip scanning protocol is to cancel out the phase background due to the retinal surface topography. Then, the phase signals within each vessel were averaged for $\Delta \varphi$, after manually segmenting the vessel lumens. Four repeated measurements at each circle were averaged. The Doppler angles were calculated according to Eq. (5), and so were the velocities according to Eq. (3). Finally, the blood flow $F(\mu \mathrm{L} / \min )$ was calculated as

$F=\sin \theta_{1} \times v \times \frac{\pi}{4} \times D^{2}$,

where $D(\mu \mathrm{m})$ is the diameter of the vessel which was calculated by manual segmentation of the vessel lumen area.

\subsection{RNFL Thickness Measurements}

Inner circular scanning data from the visible light channel were used for measuring RNFL thickness, for better axial resolution. We used circular scanning as it has much higher sampling density, better signal-to-noise ratio, and is also consistent with current clinical protocols. Figure 2(e) shows the identification of RNFL in B-scan images. By using visible light channel for better axial resolution, we identified the RNFL by the bright reflectance signal and used Image $J$ to measure the RNFL thickness manually. We used multiple measurements $(\geq 5)$ at different radial locations and then averaged them to obtain the value for each retina. The measurements were repeated three times, and there was no statistical difference among different measurements.

\subsection{Spectroscopic Marker VN Ratio}

The method to calculate VN ratio has been described in our previous publication. ${ }^{21}$ The $\mathrm{VN}$ ratio quantifies the spectroscopic contrast between the visible and NIR channels and thus allows ultrastructural measurements beyond the resolution limit of current NIR OCT. The VN ratio is calculated based on the absolute image intensity of OCT images in the visible and NIR channels on a linear scale. Due to the transparency of the aqueous humor, the signal floor above the retina reflects the systemic noise due to the reference signal and electronic noise. Therefore, the noise floor calculated above the retina in the image was subtracted in both the visible and NIR channels.

To calculate VN ratio, we first interpolated the NIR cross sectional (B-scan) image in the axial dimension for a consistent scale to the visible B-scan image and coregistered the two images by shifting the NIR B-scan image. The surface of the inner retina was detected by setting an intensity threshold. The intensity from the superficial $30 \mu \mathrm{m}$ was integrated in each channel, and the ratio between visible over NIR channel produced the VN ratio. The choice of $30 \mu \mathrm{m}$ is to include signals primarily from RNFL and RGCs while ensuring sufficient thickness for the signal integration. We performed manual image segmentations on the en face OCT projections to select regions of interest (ROI) and to avoid blood vessels. The VN ratios from all ROIs were averaged to represent the reading for one retina.

To ensure the measurements were free of systemic variation, we used the $\mathrm{VN}$ ratio from blood as an on-site in vivo reference because we considered the optical properties of blood are constant. The VN ratio by integrating a $50 \mu$ m thickness of whole blood is theoretically calculated to be $\sim 0.6$ with $<10 \%$ variation between oxygenated and deoxygenated blood. ${ }^{21}$ Then, the VN ratio from RNFL/RGCs is adjusted as

$\mathrm{VN}=\frac{\mathrm{VN}_{\text {raw }}}{\mathrm{VN}_{\text {blood }}} \times 0.6$.

\subsection{Dexamethasone-Induced Ocular Hypertension Mouse Model}

An established dexamethasone-induced ocular hypertension mouse model was used in this study. ${ }^{35-37}$ Ten-week-old C57BL/6 male mice were purchased from Jackson lab. IOP elevation was achieved by treating the eyes with $0.1 \%$ dexamethasone eye drop twice daily for 7 weeks. The control group mice received saline eye drops twice daily. The IOP from each eye was measured weekly by a tonometer until the termination of this study. For eye drop administration and IOP measurements, animals were placed in the induction chamber for $\sim 2$ min under $3 \%$ isoflurane carried by $1.5 \mathrm{~L} / \mathrm{min}$ oxygen flow. Then, the animal was placed on a stage, while $1.5 \%$ isoflurane was delivered by a nose cone. The IOP measurements were performed within $3 \mathrm{~min}$ of induction to avoid the influence of anesthesia on IOP. ${ }^{38}$

\subsection{Eye Dissection and Immunohistochemistry}

At the end point of the study (7 weeks), mice were euthanized and their eyes were enucleated using a curved tip tweezer. The retinas were subsequently dissected, and fixed in $-20^{\circ} \mathrm{C}$ methanol. For immunofluorescence staining, the retinas were first rinsed in PBS to wash away the methanol, and then were washed 
(a)

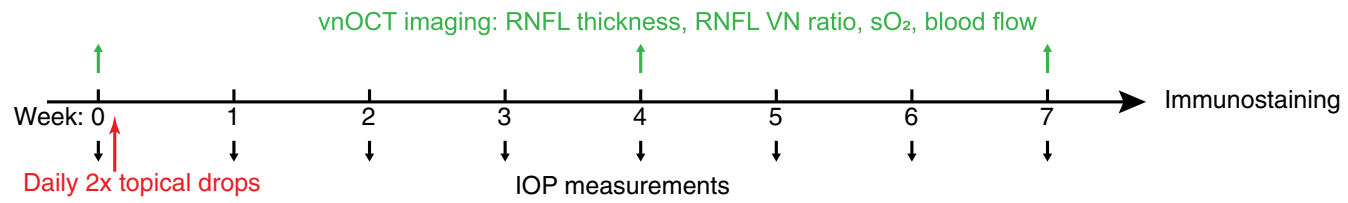

(b)

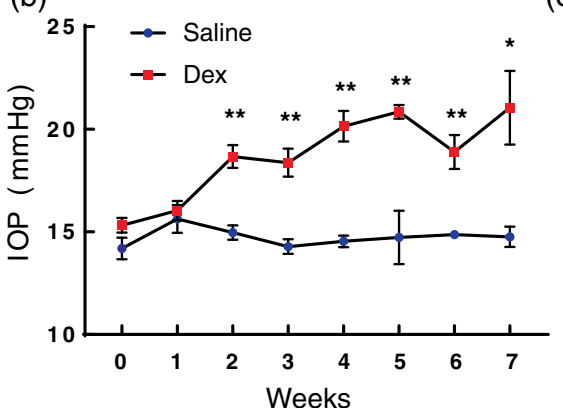

(c)

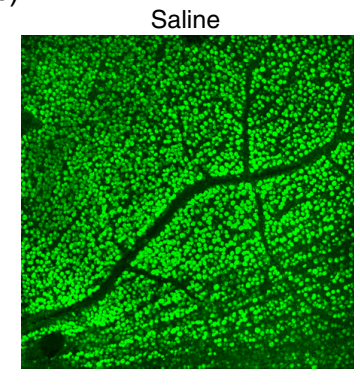

(d)

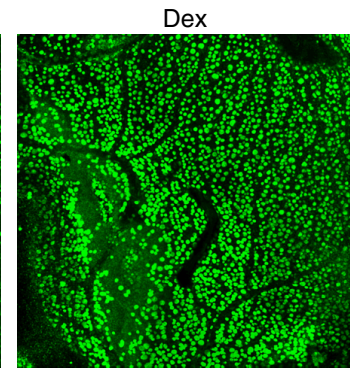

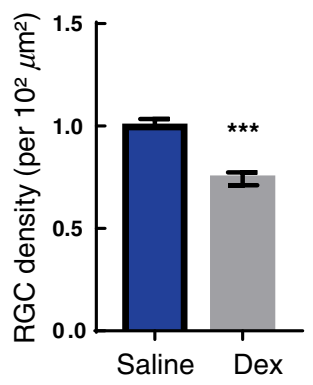

Fig. 3 Characterization of the dexamethasone-induced ocular hypertension mouse model. (a) The timeline of measurements over the span of 7 weeks. (b) Weekly measurements of IOP by a tonometer. (c) Representative confocal fluorescence microscopic images of anti-Brn3a immunostaining for RGCs, in the saline and dexamethasone groups. (d) Comparison of the RGC density between saline $(n=8)$ and dexamethasone groups $(n=8) . * p<0.05, * * p<0.01, * * * p<0.001$. Error bar $=\mathrm{SEM}$.

(a)

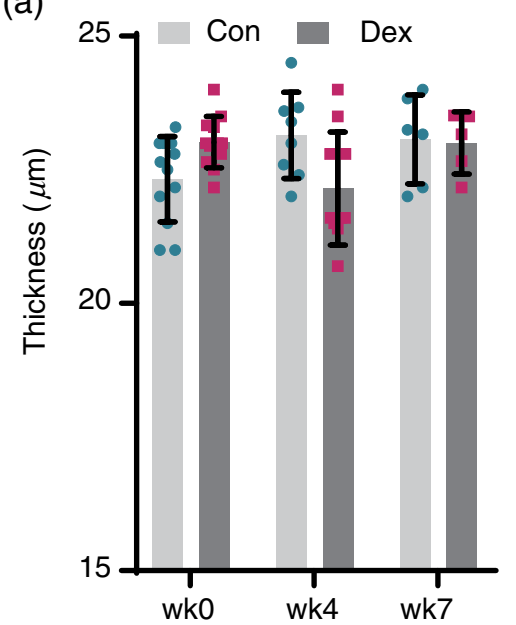

(d)

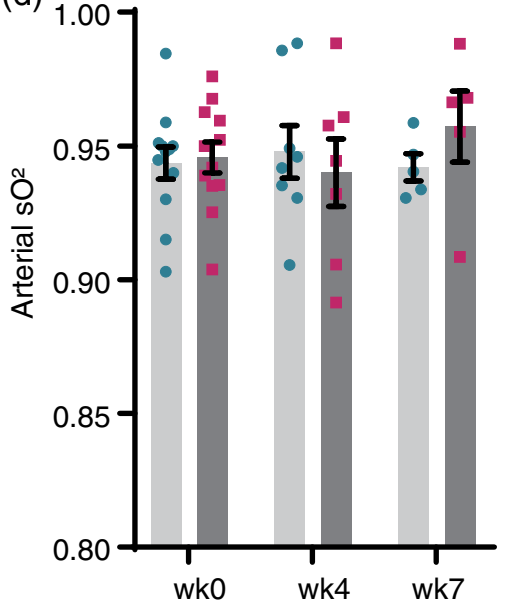

(b)

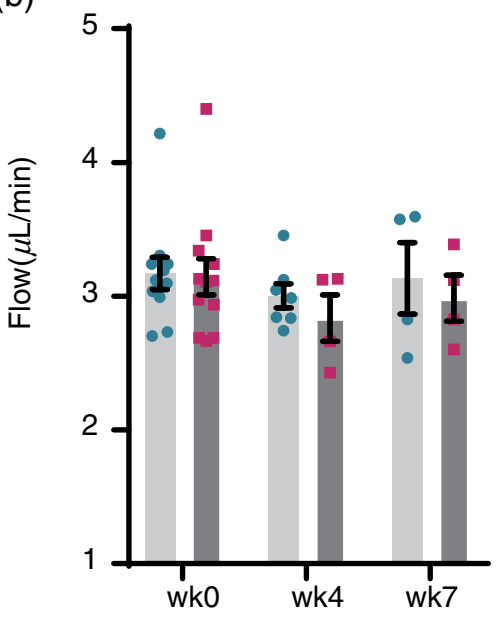

(e)

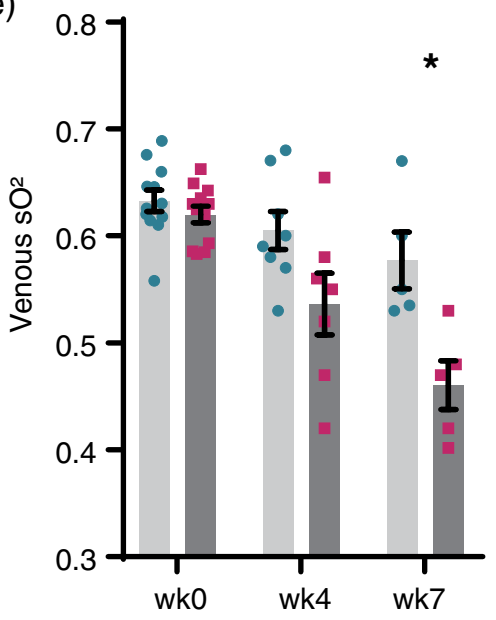

(c)

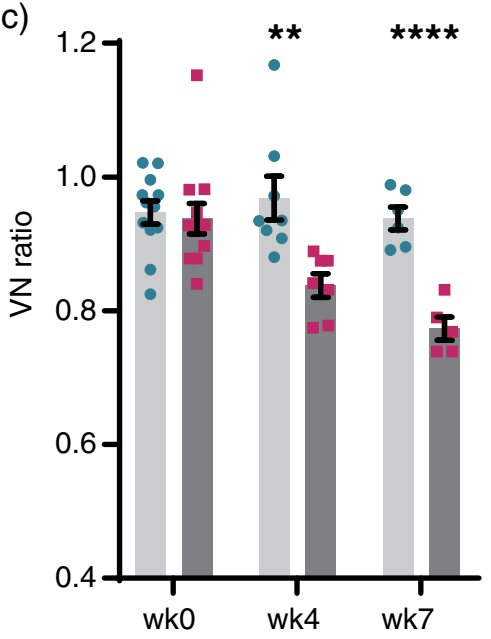

(f)

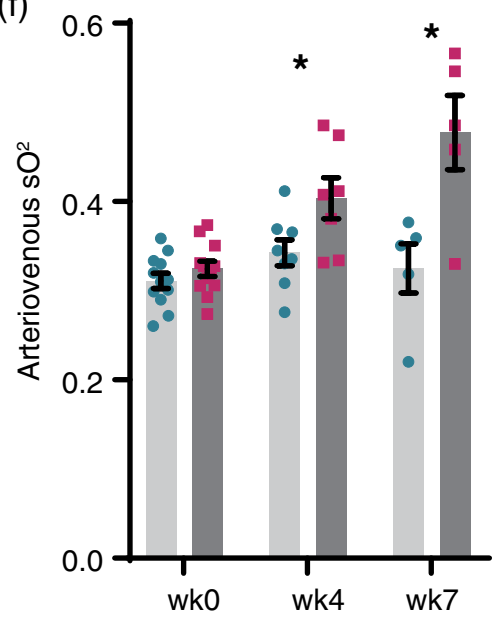

Fig. 4 Longitudinal measurements by vnOCT at weeks 0 , 4, and 7 for (a) RNFL thickness ( $\mu \mathrm{m}$ ), (b) blood flow ( $\mu \mathrm{L} / \mathrm{min}$ ), (c) $\mathrm{VN}$ ratio, (d) arterial $\mathrm{sO}_{2}$, (e) venous $\mathrm{sO}_{2}$, and (f) arteriovenous(A-V) $\mathrm{sO}_{2}$ difference. $* P<0.05 ; * * P<0.01 ; * * * * P<0.0001$. Error bar $=\mathrm{SEM}$. 
three times (10 min each) in $0.05 \%$ PBST $(0.05 \%$ Triton X-100 PBS solution). After that, the retinas were blocked/permeabilized for $1.5 \mathrm{~h}$ at room temperature in a solution containing $0.25 \%$ Triton X-100 and 10\% goat serum in PBS. Immunostaining was performed using primary and secondary antibodies. The primary antibody, rabbit anti-Brn3a (Millipore Sigma) was diluted 1:500 in 5\% goat serum in PBS and incubated with the retinas overnight at $4^{\circ} \mathrm{C}$. After three washes in $0.05 \%$ PBST, the retinas were incubated for an additional $2 \mathrm{~h}$ at room temperature with Alexa Fluor 488 conjugated goat anti-rabbit secondary antibody (Invitrogen) diluted (1:800) in 10\% goat serum in PBS. Finally, the retinas were rinsed two times with PBS, mounted in aqueous mounting medium, and cover-slipped for imaging.

\subsection{Cell Counting}

After immunofluorescence staining, the whole mount retina slides were imaged by a confocal fluorescence microscope (Leica SP5, 10× objective). The FOV of each image is $0.78^{2} \mathrm{~mm}^{2}$. The depth of focus is $8 \mu \mathrm{m}$. Due to the significant shrinking after fixation and thin layer of RGCs, the depth of focus allowed capturing local RGCs immunofluorescence signals. The number of stained RGCs within an ROI avoiding large vessels and nonuniform areas of immunostaining was manually counted from the confocal images in Image J. The RGC cell density was then calculated by the cell number per ROI area. At least four ROIs from each retina image were used with the average cell density representing the reading from each retina. The measurements were repeated twice, and there was no significant difference.

\subsection{Statistical Analysis}

Two sample Student's $T$-test was used in Figs. 3 and 4. The Pearson's linear regression was used to test the correlation among IOP, VN ratio, and $\mathrm{sO}_{2}$ in Fig. 5.

\section{Results}

The study design is shown in Fig. 3(a). IOP measurements were performed weekly to confirm the induced ocular hypertension. The vnOCT imaging was performed longitudinally at the baseline week 0 , week 4 , and week 7 . The study was terminated at 7 weeks, and the effectiveness of the model was further verified by measuring RGC loss via immunostaining and cell counting. The study started with 12 animals, six in the dexamethasone group and six in the control group. Four animals from each group reached the terminal stage at week 7 (Table 1).

The animal model was first validated by measuring IOP and then RGC counting. Baseline IOP was noted to be $14.2 \pm 1.7$ and $15.3 \pm 1.2$ for both the control and dexamethasone group, respectively, and this was not statistically different. The IOP in the dexamethasone group showed a statistically significant increase as compared to the control group by week $2[p<0.05$, Fig. 3(b)]. The characteristics of the IOP change are consistent with multiple previous publications using this animal model. ${ }^{36-38}$ The RGC density (cells per $10^{2} \mu \mathrm{m}^{2}$ ) as measured by confocal fluorescence microscopic images at week 7 [Figs. 3(c) and 3(d)] was significantly decreased in the dexamethasone group compared to the control group $[p=0.0002$, Fig. 3(e)], confirming the RGC loss.

The results of longitudinal multimetric measurements by vnOCT are summarized in Fig. 4 and Table 2. The RNFL (a)

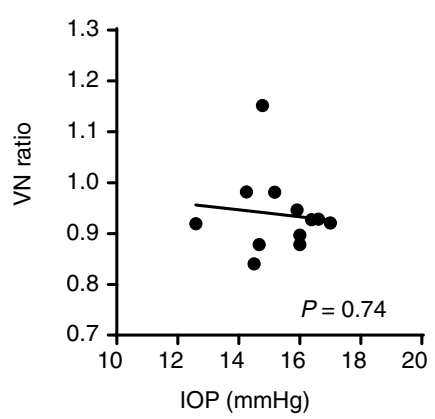

(b)
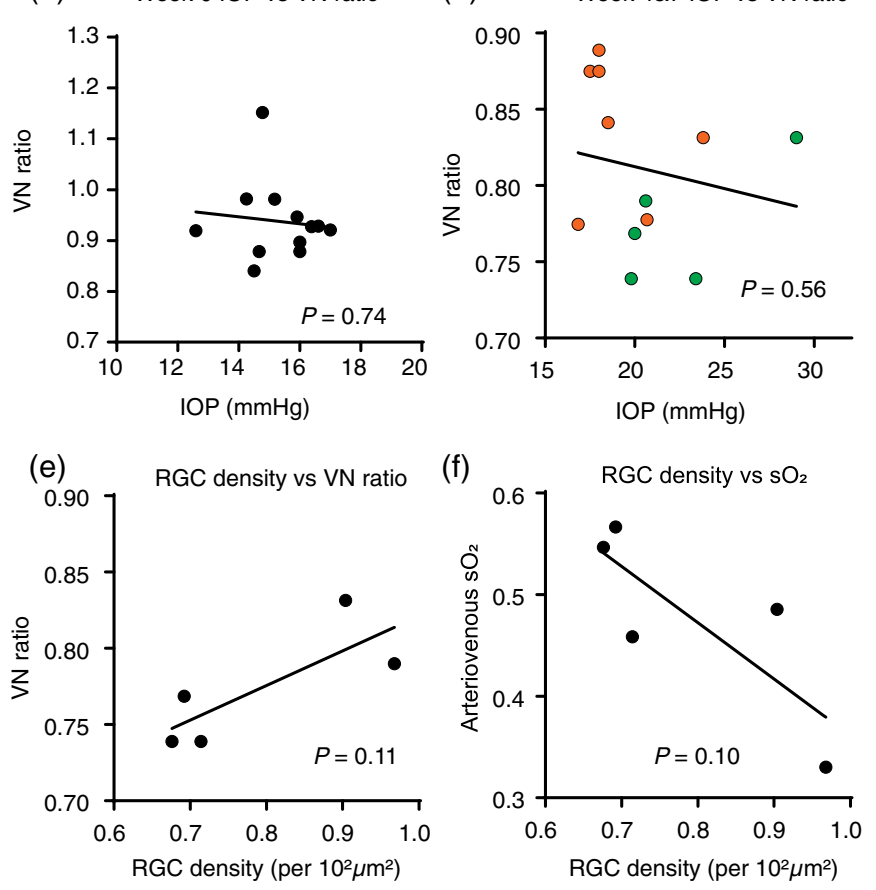

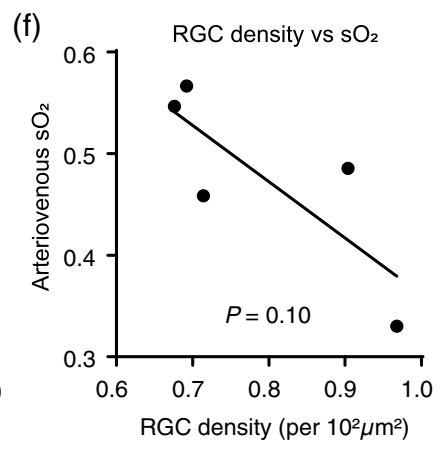

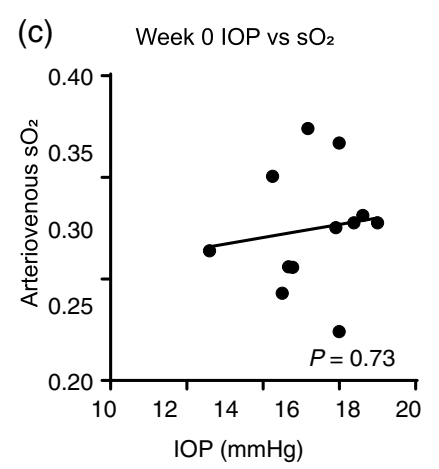
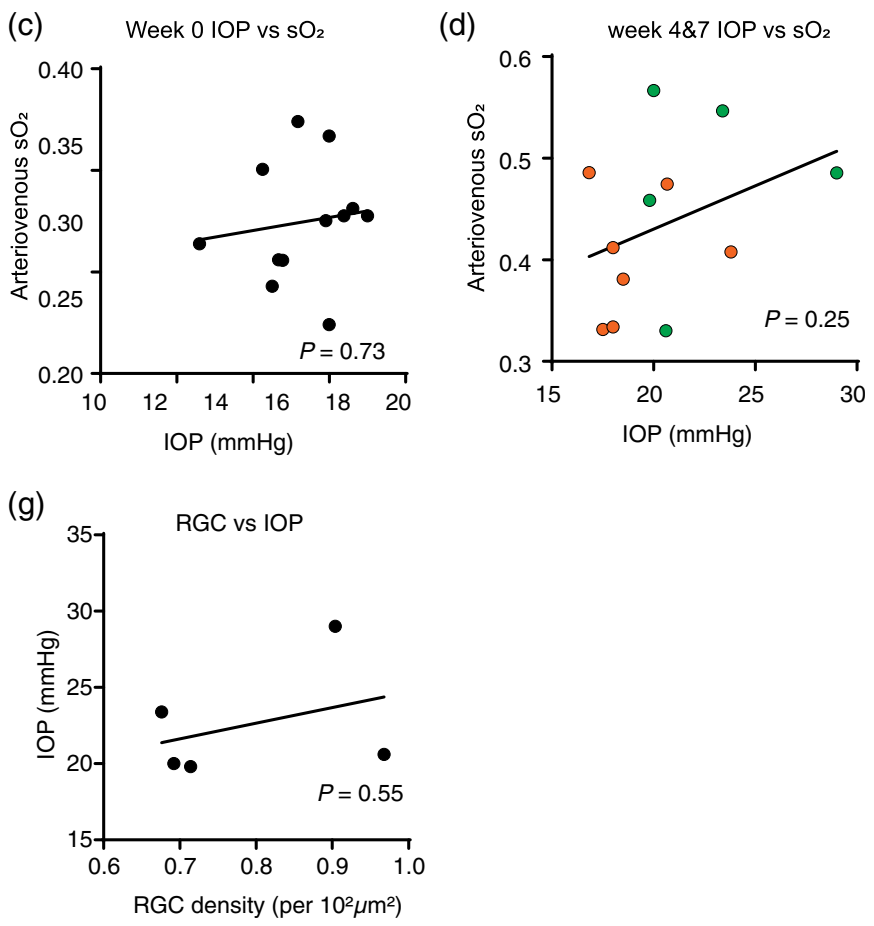

Fig. 5 Correlation among vnOCT markers, IOP and RGC density in the dexamethasone-treated group. (a), (b) The correlation between VN ratio and IOP in each eye in the dexamethasone group at week 0 and weeks 4 and 7. (c), (d) The correlation between $\mathrm{A}-\mathrm{V} \mathrm{sO}_{2}$ difference and IOP in each eye in the dexamethasone group at week 0 and weeks 4 and 7. (e)-(g) The correlation between $\mathrm{VN}$ ratio, $\mathrm{A}-\mathrm{V} \mathrm{sO}_{2}$ difference, IOP and RGC density in each eye in the dexamethasone group at terminal week 7. 
Table 1 Summary of IOP $(\mathrm{mmHg})$ for individual retinas.

\begin{tabular}{lcccccccc}
\multicolumn{8}{c}{ Control group } \\
\hline Eye ID & Wk0 & Wk1 & Wk2 & Wk3 & Wk4 & Wk5 & Wk6 & Wk7 \\
\hline 1OS & 13.67 & 13.31 & 14.88 & 14.50 & 15.25 & 7.50 & 14.40 & 13.83 \\
1OD & 14.67 & 15.20 & 14.79 & 14.88 & 14.00 & 14.67 & 14.60 & 15.80 \\
2OS & 14.14 & & & & & & & \\
2OD & 12.00 & & & & & & & \\
3OS & 15.75 & 19.17 & 16.29 & 15.06 & 15.20 & 15.80 & 14.67 & 12.80 \\
3OD & 14.14 & 17.53 & 16.50 & 15.08 & 14.20 & 20.00 & 15.00 & 13.20 \\
$4 O S$ & 12.14 & 14.00 & 15.13 & 13.67 & 12.80 & 12.20 & 14.00 & 15.40 \\
$4 O D$ & 12.33 & 15.40 & 14.00 & 13.25 & 14.20 & 15.00 & 14.83 & 14.67 \\
$5 O S$ & 12.71 & & & & & & & \\
$5 O D$ & 15.14 & & & & & & & \\
6 OS & 14.50 & 15.80 & 13.71 & 12.57 & 15.17 & 15.50 & 15.60 & 15.60 \\
$6 O D$ & 15.00 & 14.60 & 14.44 & 15.27 & 15.40 & 17.20 & 15.80 & 16.80
\end{tabular}

\begin{tabular}{|c|c|c|c|c|c|c|c|c|}
\hline \multicolumn{9}{|c|}{ Dexamethasone group } \\
\hline Eye ID & WkO & Wk1 & Wk2 & Wk3 & Wk4 & Wk5 & Wk6 & Wk7 \\
\hline $70 s$ & 16.00 & 18.20 & 17.73 & 16.33 & 16.83 & & & \\
\hline $7 O D$ & 16.38 & 15.17 & 22.00 & 17.00 & 17.50 & & & \\
\hline $80 S$ & 15.18 & 16.80 & 18.17 & 19.11 & 23.80 & 19.80 & 17.80 & 20.60 \\
\hline $80 D$ & 14.78 & 13.75 & 21.38 & 20.71 & 20.67 & 21.20 & 19.00 & 11.00 \\
\hline $90 \mathrm{~s}$ & 14.50 & 13.50 & 16.00 & 24.50 & 20.00 & 22.40 & 13.75 & \\
\hline $9 O D$ & 15.91 & 16.40 & 16.00 & 19.19 & 16.60 & 20.00 & 16.00 & \\
\hline $100 S$ & 14.67 & 15.50 & 16.22 & 17.50 & 18.50 & 20.40 & 20.00 & 20.00 \\
\hline $100 D$ & 12.60 & 16.89 & 18.77 & 16.83 & 18.00 & 21.33 & 20.00 & 19.80 \\
\hline $110 \mathrm{~S}$ & 14.25 & 17.83 & 18.67 & 16.00 & 18.00 & 22.00 & 21.00 & 20.80 \\
\hline $110 D$ & 16.62 & 14.14 & 17.93 & 18.73 & 22.33 & 19.20 & 18.00 & 23.83 \\
\hline $120 S$ & 17.00 & 16.43 & 19.25 & 17.57 & 22.60 & 20.40 & 20.80 & 23.40 \\
\hline $12 \mathrm{OD}$ & 16.00 & 17.89 & 19.26 & 17.00 & 21.00 & 21.80 & 22.60 & 29.00 \\
\hline
\end{tabular}

Note: Bold font, no data loss; empty cells, animal expired. Mouse \#2, 5,7 did not recover from anesthesia after vnOCT imaging. Mouse \#9 did not recover anesthesia after dexamethasone treatment; italic font, vnOCT data were excluded due to poor image quality. The number in the table is IOP.

thickness was similar at baseline between the two groups and did not change significantly from baseline to week 4 or 7 for either group, or comparatively [Fig. 4(a)]. Blood flow measurements were similar at baseline and showed only a moderate decrease when comparing the dexamethasone to control group at weeks $4(p=0.36)$ and $7(p=0.65)$ [Fig. 4(b)]. Baseline
RNFL-RGC VN ratio measurements were similar between the two groups, but decreased progressively at week 4 ( $p=0.0048)$ and $7(p=0.00009)$ in the dexamethasone group compared to the control group [Fig. 4(c)]. This change is consistent with the previous reported spectroscopic change, which was attributed to the ultrastructural cytoskeleton loss in RGCs. ${ }^{24}$

The retinal arterial and venous $\mathrm{sO}_{2}$ were similar at baseline between the two groups. The arterial $\mathrm{sO}_{2}$ did not show any statistically significant change temporally or comparatively between the groups throughout the study. The venous $\mathrm{sO}_{2}$ did show a progressive drop in the dexamethasone group compared to the control, which was statistically significant at week $7\left[p=0.01\right.$, Fig. 4(e)]. As a result, the A-V sO ${ }_{2}$ also showed a progressive increase at week $4(p=0.039)$ and week $7(p=0.016)$, indicating a higher oxygen extraction efficiency from the inner retinal circulation in the dexamethasone versus control group [Fig. 4(f)].

Since the vnOCT measurements showed that VN ratio and $\mathrm{A}-\mathrm{V} \mathrm{sO}_{2}$ were significantly altered in dexamethasone group, we next examined the correlation of these two markers to IOP and RGC loss. We first compared $\mathrm{VN}$ ratio, and $\mathrm{A}-\mathrm{V} \mathrm{s} \mathrm{s}_{2}$ with IOP at weeks 0 and week 4 and 7 in dexamethasone-treated ocular hypertensive eyes [Figs. 5(a)-5(d)] and found only weak correlation by Pearson's test. We next compared RGC density measurement with the terminal measurement of $\mathrm{VN}$ ratio, $\mathrm{A}-\mathrm{V}$ $\mathrm{sO}_{2}$ difference, and IOP at week 7 [Figs. 5(e)-5(g)]. Although the sample number is small, there appears to be moderate correlation between $\mathrm{VN}$ ratio, $\mathrm{A}-\mathrm{V} \mathrm{sO} 2, \mathrm{IOP}$, and $\mathrm{RGC}$ loss. This analysis suggests that $\mathrm{VN}$ ratio and $\mathrm{A}-\mathrm{V} \mathrm{sO}_{2}$ may effectively serve as markers for RGC damage, potentially independent of IOP.

\section{Discussion}

In this study, we demonstrated that spectroscopic markers (VN ratio and $\mathrm{A}-\mathrm{V} \mathrm{sO}_{2}$ ) enabled by vnOCT have the potential to detect $\mathrm{VN}$ ratio changes in the RNFL-RGC and alterations in blood hemoglobin $\mathrm{sO}_{2}$ within the inner retinal circulation associated with ocular hypertension in a mouse model. These findings suggest that a decreased $\mathrm{VN}$ ratio and an increased $\mathrm{A}-\mathrm{V} \mathrm{sO}_{2}$ difference may represent early changes in the development of glaucoma.

One key measurement enabled by vnOCT is RNFL-RGC VN ratio, which quantifies the spectral contrast between visible and NIR channels. The image intensities of visible and/or NIR band can be variable across retinas and within retina, affected by the transmission from anterior part of the eye, angle of the laser passing through the pupil, and the eye quality (dry or moist, eye condition etc.). To control those experimental variables, we have used the circulating whole blood as an in situ spectral reference to calibrate systemic variations and used the mean $\mathrm{VN}$ ratio to represent the whole retina. The VN ratio calculated from different thickness from the surface of the retina is shown as Fig. 6. It shows that the calculation tends to stabilize after 25 to $30 \mu \mathrm{m}$ thickness, and the separation between ocular hypertensive and control eyes was still significant. Therefore, we used $30 \mu \mathrm{m}$ for all our calculation, for consistency. Although the superficial $30 \mu \mathrm{m}$ tissue also includes some signals from RGCs, the depth selection was a balance for the measurement robustness. We also took total blood flow, mean $\mathrm{sO}_{2}$ over arterials and venules, and mean RNFL thickness to represent each retina in this study.

The dexamethasone mouse is a well-established model of steroid-induced ocular hypertension and glaucoma. ${ }^{36-38}$ 
Table 2 Summary for longitudinal measurements by vnOCT.

\begin{tabular}{|c|c|c|c|c|}
\hline \multirow[b]{2}{*}{ Parameters } & & \multirow{2}{*}{$\begin{array}{c}\text { Week } 0 \\
\text { Mean } \pm \text { SEM }\end{array}$} & \multirow{2}{*}{$\frac{\text { Week } 4}{\text { Mean } \pm \text { SEM }}$} & \multirow{2}{*}{$\begin{array}{c}\text { Week } 7 \\
\text { Mean } \pm \text { SEM }\end{array}$} \\
\hline & & & & \\
\hline \multirow[t]{2}{*}{ Thickness $(\mu \mathrm{m})$} & CON & $22.33 \pm 0.22$ & $23.15 \pm 0.27$ & $23.07 \pm 0.31$ \\
\hline & DEX & $23.02 \pm 0.13$ & $22.15 \pm 0.32$ & $23.17 \pm 0.25$ \\
\hline \multirow[t]{2}{*}{ Flow $(\mu \mathrm{L} / \mathrm{min})$} & $\mathrm{CON}$ & $3.17 \pm 0.12$ & $3.00 \pm 0.09$ & $3.13 \pm 0.28$ \\
\hline & DEX & $3.14 \pm 0.13$ & $2.97 \pm 0.14$ & $2.98 \pm 0.17$ \\
\hline \multirow[t]{2}{*}{ VN ratio (uniless) } & CON & $0.95 \pm 0.02$ & $0.97 \pm 0.03$ & $0.94 \pm 0.02$ \\
\hline & DEX & $0.94 \pm 0.02$ & $\mathbf{0 . 8 4} \pm 0.02$ & $\mathbf{0 . 7 7} \pm 0.02$ \\
\hline \multirow{2}{*}{ Arterial $\mathrm{sO}_{2}(100 \%)$} & CON & $0.9437 \pm 0.0057$ & $0.9478 \pm 0.0092$ & $0.9420 \pm 0.0045$ \\
\hline & DEX & $0.9457 \pm 0.0055$ & $0.9401 \pm 0.0117$ & $0.9573 \pm 0.0119$ \\
\hline \multirow[t]{2}{*}{ Venous $\mathrm{sO}_{2}(100 \%)$} & CON & $0.6326 \pm 0.0095$ & $0.6050 \pm 0.0167$ & $0.5770 \pm 0.0236$ \\
\hline & DEX & $0.6199 \pm 0.0075$ & $0.5363 \pm 0.0266$ & $0.4603 \pm 0.0204$ \\
\hline \multirow[t]{2}{*}{$\mathrm{AV} \mathrm{sO} \mathrm{S}_{2}(100 \%)$} & CON & $0.3110 \pm 0.0080$ & $\mathbf{0 . 3 4 2 8} \pm 0.0136$ & $0.3251 \pm 0.0248$ \\
\hline & DEX & $\mathbf{0 . 3 2 4 6} \pm 0.0080$ & $0.4037 \pm 0.0214$ & $\mathbf{0 . 4 7 7 3} \pm 0.0373$ \\
\hline
\end{tabular}

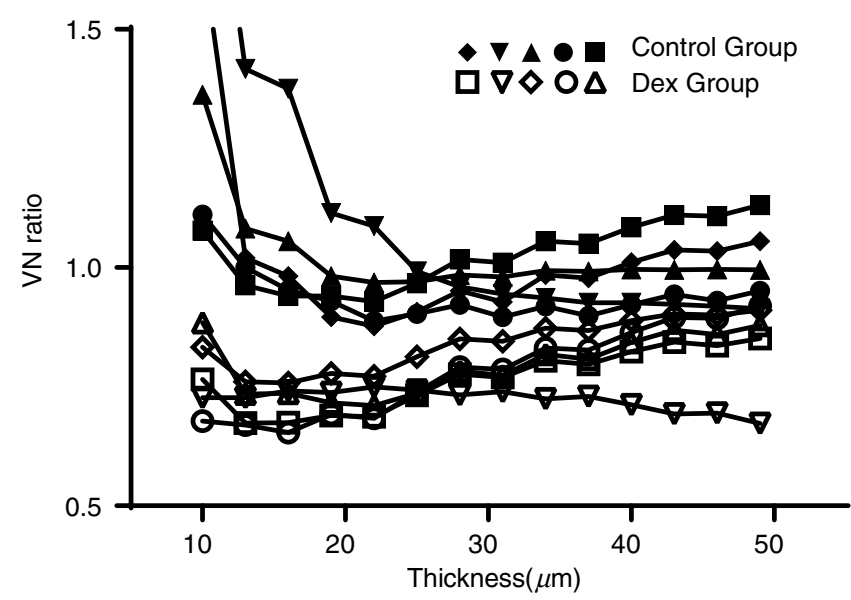

Fig. 6 VN ratio calculated from varying thickness from the retinal surface at week 7 . Solid and blank symbols labeled the eyes in control and Dex groups, respectively.

Consistent with previous reports, our mice developed significantly increased IOP within 2 weeks of starting topical dexamethasone drops with subsequent progression to RGC loss. The mechanism by which steroids cause ocular hypertension remains to be fully elucidated but decreased outflow facility is considered the main contributing factor. Phulke et al. ${ }^{39}$ have nicely outlined the leading molecular pathways to reduced outflow facility including accumulation of glycosaminoglycans, laminin, elastin, and fibronectin in the trabecular meshwork (TM), increased TM cell nuclear size and DNA content, and decreased prostaglandin synthesis. ${ }^{39-43}$ Steroids may also upregulate myocylin gene (MYOC/GLC1A) expression, accumulation of which is an initial step in the development of glaucoma. ${ }^{44,45}$ A number of other genes are thought to contribute as well, though their significance is still being evaluated. ${ }^{46-48}$ The resulting elevated IOP is transmitted posteriorly inducing a cascade of events that ultimately cause RGC axonal damage followed by RGC loss, mimicking the major pathologic events of primary open angle glaucoma.

While our study found an early elevation in IOP and resulting RGC loss, it did not result in significant change to the RNFL thickness within the first 7 weeks in our study. This finding/ result corroborates with other preclinical studies having shown that RGC loss precedes RNFL thickness change following acute optic nerve injury. ${ }^{49,50}$ The VN ratio, however, progressively decreased over this time period, suggesting that ultrastructural changes may precede RNFL loss in early glaucoma. As the VN ratio is derived from the superficial $30 \mu \mathrm{m}$ of RNFL-RGC around the $\mathrm{ONH}$, it primarily represents signal from RGC axons, in which microtubules contribute a significant portion of the measured reflectance. ${ }^{51}$ Therefore, the decreased VN ratio may reflect ocular hypertension-induced structural alterations in the RGC cytoskeletons. ${ }^{52,53}$ Indeed, previous ex vivo studies have established that the cytoskeletal alterations in RNFL axons is an early event of RGC damage, resulting a "flatter" reflectance spectrum from RNFL, detectable prior to RNFL thinning. ${ }^{24}$ This altered reflectance spectrum corresponds to our findings of a decreased VN ratio, further supporting our hypothesis that the decreased VN ratio may be a sensitive ultrastructural optical marker for early detection of RGC damage. One discrepancy between our study and the previous study is that ex vivo retinas have $\mathrm{VN}$ ratio larger than 1 while we found $\mathrm{VN}$ ratio slightly less than 1 from in vivo retinas when we compared visible and NIR bands centered at 560 and $820 \mathrm{~nm}$. The study by $\mathrm{Yi}$ et al. ${ }^{54}$ used fixed ex vivo retinas and only investigated changes in visible light range by confocal reflectance microscopy. The study by Huang et al. ${ }^{24}$ used different device and normalization methods for spectral characterization and 
used a different ex vivo preparation for retina culturing. Those differences are all likely to cause the discrepancy.

Alterations in the retinal circulation are considered important events in glaucoma development. ${ }^{55}$ Lower ocular perfusion pressure and low systolic and diastolic blood pressure are known to be major risk factors for glaucoma. ${ }^{56}$ Numerous studies have consistently shown the reduced blood flow in glaucomatous eyes. ${ }^{14,57-59}$ Recent OCTA studies have shown that the peripapillary capillary vasculature density and capillary flow index are compromised in advanced glaucomatous eyes, ${ }^{10-15}$ suggesting microvascular perfusion around the $\mathrm{ONH}$ is an important factor in glaucoma's pathogenesis. As retinal function relies on oxygen metabolism, changes in blood $\mathrm{sO}_{2}$ represent an attractive potential indicator of functional impairment. In our study, blood flow shows only a moderate decrease in the dexamethasone group compared to control group at weeks 4 and 7 . The increased arteriovenous $\mathrm{sO}_{2}$ difference $\left(\mathrm{A}-\mathrm{V} \mathrm{sO}_{2}\right)$, driven by decreased venous $\mathrm{sO}_{2}$, is more significant, indicating more oxygen extraction from the retinal circulation. Even with the mild decrease in blood flow, overall oxygen consumption from the retinal circulation increases, suggesting a state of oxygen hypermetabolism. This finding is in contrast to later stages of glaucoma when $\mathrm{A}-\mathrm{V} \mathrm{sO}_{2}$ is found to be decreased due to advanced cell loss and decreased oxygen consumption. ${ }^{60}$ Our suggestion of a dynamic change in oxygen metabolism associated with glaucoma progression is consistent with previous studies of a diabetic retinopathy mouse model, in which early oxygen hypermetabolism eventually gives way to a cell death-mediated decrease in oxygen consumption. ${ }^{61}$ The underlying biology of the early hypermetabolism is not clear at this moment and requires further investigation. Nevertheless, our data herein suggest that in early stage glaucoma, global alterations of $\mathrm{sO}_{2}$ might be another sensitive marker for early glaucoma.

The major limitation of this study is the limited number of data points by week 7 , when only four animals and five eyes per group producing good quality images and vnOCT readings, thereby limiting the statistical power in Figs. 5(c) and 5(d). Several animals expired during the study and loss of corneal clarity in some eyes prevented reliable retinal imaging. It is reassuring, however, that measured changes of RNFL VN ratio and $\mathrm{AV}-\mathrm{sO}_{2}$ difference already reached statistical significance with a higher number of data points by week 4 .

\section{Conclusion}

In summary, we demonstrate that vnOCT can detect spectroscopic changes in RNFL-RGC, in addition to changes in the oxygen levels in the retinal circulation in a dexamethasoneinduced ocular hypertension mouse model. The changes were identifiable even within the short-time frame of this study, indicating that pressure elevations can result in retinal changes prior to measurable alteration of RNFL thickness. These findings pave the way for future studies in mice and humans to gain further insight as to how changes progress, if they are reversible, and how they may respond to treatment.

\section{Disclosures}

The authors have no relevant financial interests in this article and no potential conflicts of interest to disclose.

\section{Acknowledgments}

The work contained in this paper was supported by Bright Focus Foundation (G2017077, M2018133), BU-CTSI
1KL2TR001411, National Institute of Neurological Disorders and Stroke (1R01NS108464-01), National Eye Institute (1R21 EY029412), and National Cancer Institute (1R01CA22491101A1). We would like to thank Professor Haiyan Gong and Dr. Ruiyi Ren for their generous help on the dexamethasoneinduced ocular hypertension mouse model and intraocular pressure measurement.

\section{References}

1. M. V. Boland et al., Treatment for Glaucoma: Comparative Effectiveness, Agency for Healthcare Research and Quality, Rockville, Maryland (2012).

2. R. S. Harwerth et al., "Ganglion cell losses underlying visual field defects from experimental glaucoma," Invest. Ophthalmol. Visual Sci. 40, 2242-2250 (1999).

3. L. A. Kerrigan-Baumrind et al., "Number of ganglion cells in glaucoma eyes compared with threshold visual field tests in the same persons," Invest. Ophthalmol. Visual Sci. 41, 741-748 (2000).

4. M. A. Kass et al., "The ocular hypertension treatment study—a randomized trial determines that topical ocular hypotensive medication delays or prevents the onset of primary open-angle glaucoma," Arch. Ophthalmol. 120, 701-713 (2002).

5. M. O. Gordon et al., "The ocular hypertension treatment study-baseline factors that predict the onset of primary open-angle glaucoma," Arch. Ophthalmol. 120, 714-720 (2002).

6. J. S. Schuman et al., "Quantification of nerve fiber layer thickness in normal and glaucomatous eyes using optical coherence tomography: a pilot study," Arch. Ophthalmol. 113, 586-596 (1995).

7. A. Kanamori et al., "Evaluation of the glaucomatous damage on retinal nerve fiber layer thickness measured by optical coherence tomography," Am. J. Ophthalmol. 135, 513-520 (2003).

8. A. T.-H. Lu et al., "Combining nerve fiber layer parameters to optimize glaucoma diagnosis with optical coherence tomography," Ophthalmology 115, 1352-1357.e2 (2008).

9. A. L. Silverman et al., "Diagnostic accuracy of the spectralis and cirrus reference databases in differentiating between healthy and early glaucoma eyes," Ophthalmology 123, 408-414 (2016).

10. L. S. Geyman et al., "Peripapillary perfused capillary density in primary open-angle glaucoma across disease stage: an optical coherence tomography angiography study," Br. J. Ophthalmol. 101, 1261-1268 (2017).

11. Z. Mammo et al., "Quantitative optical coherence tomography angiography of radial peripapillary capillaries in glaucoma, glaucoma suspect, and normal eyes," Am. J. Ophthalmol. 170, $41-49$ (2016).

12. S. H. Zhang et al., "Optical coherence tomography angiography of the peripapillary retina in primary angle-closure glaucoma," Am. J. Ophthalmol. 182, 194-200 (2017).

13. L. Liu et al., "Projection-resolved optical coherence tomography angiography of the peripapillary retina in glaucoma," Am. J. Ophthalmol. (2019).

14. L. Liu et al., "Optical coherence tomography angiography of the peripapillary retina in glaucoma," JAMA Ophthalmol. 133, 1045-1052 (2015).

15. R. Mastropasqua et al., "Optical coherence tomography angiography of the peripapillary retina in normal-tension glaucoma and chronic nonarteritic anterior ischemic optic neuropathy," Curr. Eye Res. 43, 778-784 (2018).

16. J. Yi et al., "Visible-light optical coherence tomography for retinal oximetry," Opt. Lett. 38, 1796-1798 (2013).

17. J. Yi et al., "Human retinal imaging using visible-light optical coherence tomography guided by scanning laser ophthalmoscopy," Biomed. Opt. Express 6, 3701-3713 (2015).

18. X. Shu, L. J. Beckmann, and H. F. Zhang, "Visible-light optical coherence tomography: a review," J. Biomed. Opt. 22(12), 121707 (2017).

19. J. Yi et al., "Visible light optical coherence tomography measures retinal oxygen metabolic response to systemic oxygenation," Light Sci. Appl. 4, e334 (2015)

20. A. J. Radosevich et al., "Structural length-scale sensitivities of reflectance measurements in continuous random media under the Born approximation," Opt. Lett. 37, 5220-5222 (2012). 
21. W. Song et al., "Fiber-based visible and near infrared optical coherence tomography (vnOCT) enables quantitative elastic light scattering spectroscopy in human retina," Biomed. Opt. Express 9, 3464-3480 (2018).

22. J. Yi et al., "Can OCT be sensitive to nanoscale structural alterations in biological tissue?" Opt. Express 21, 9043-9059 (2013).

23. X.-R. Huang et al., "Reflectance decreases before thickness changes in the retinal nerve fiber layer in glaucomatous retinas," Invest. Ophthalmol. Visual Sci. 52, 6737-6742 (2011).

24. X.-R. Huang et al., "Wavelength-dependent change of retinal nerve fiber layer reflectance in glaucomatous retinas," Invest. Ophthalmol. Visual Sci. 53, 5869-5876 (2012).

25. S. P. Chong et al., "Ultrahigh resolution retinal imaging by visible light OCT with longitudinal achromatization," Biomed. Opt. Express 9, 1477-1491 (2018).

26. J. Yi and V. Backman, "Imaging a full set of optical scattering properties of biological tissue by inverse spectroscopic optical coherence tomography," Opt. Lett. 37, 4443-4445 (2012).

27. D. J. Faber et al., "Oxygen saturation-dependent absorption and scattering of blood," Phys. Rev. Lett. 93, 028102 (2004).

28. S. Yazdanfar, A. M. Rollins, and J. A. Izatt, "Imaging and velocimetry of the human retinal circulation with color Doppler optical coherence tomography," Opt. Lett. 25, 1448-1450 (2000).

29. R. A. Leitgeb et al., "Real-time assessment of retinal blood flow with ultrafast acquisition by color Doppler Fourier domain optical coherence tomography," Opt. Express 11, 3116-3121 (2003).

30. R. A. Leitgeb et al., "Real-time measurement of in vitro flow by Fourierdomain color Doppler optical coherence tomography," Opt. Lett. 29, 171-173 (2004).

31. Y. Wang et al., "Retinal blood flow measurement by circumpapillary Fourier domain Doppler optical coherence tomography," J. Biomed. Opt. 13, 064003 (2008).

32. S. Makita et al., "Comprehensive in vivo micro-vascular imaging of the human eye by dual-beam-scan Doppler optical coherence angiography," Opt. Express 19, 1271-1283 (2011).

33. C. Dai et al., "Absolute retinal blood flow measurement with a dualbeam Doppler optical coherence tomography," Invest. Ophthalmol. Visual Sci. 54, 7998-8003 (2013).

34. W. Liu et al., "Measuring retinal blood flow in rats using Doppler optical coherence tomography without knowing eyeball axial length," Med. Phys. 42, 5356-5362 (2015).

35. D. R. Overby et al., "Ultrastructural changes associated with dexamethasone-induced ocular hypertension in mice," Invest. Ophthalmol. Visual Sci. 55, 4922-4933 (2014).

36. N. A. Whitlock et al., "Increased intraocular pressure in mice treated with dexamethasone," Invest. Ophthalmol. Visual Sci. 51, 6496-6503 (2010).

37. G. S. Zode et al., "Ocular-specific ER stress reduction rescues glaucoma in murine glucocorticoid-induced glaucoma," J. Clin. Invest. 124, 19561965 (2014).

38. C. Ding, P. Wang, and N. Tian, "Effect of general anesthetics on IOP in elevated IOP mouse model," Exp. Eye Res. 92(6), 512-520 (2011).

39. S. Phulke et al., "Steroid-induced glaucoma: an avoidable irreversible blindness," J. Curr. Glaucoma Pract. 11, 67-72 (2017).

40. J. W. Rohen, E. Linner, and R. Witmer, "Electron microscopic studies on the trabecular meshwork in two cases of corticosteroid-glaucoma," Exp. Eye Res. 17, 19-31 (1973).

41. D. H. Johnson, J. M. Bradley, and T. S. Acott, "The effect of dexamethasone on glycosaminoglycans of human trabecular meshwork in perfusion organ culture," Invest. Ophthalmol. Visual Sci. 31, 2568-2571 (1990).
42. H. T. Steely et al., "The effects of dexamethasone on fibronectin expression in cultured human trabecular meshwork cells," Invest. Ophthalmol. Visual Sci. 33, 2242-2250 (1992).

43. X. Zhang, A. F. Clark, and T. Yorio, "FK506-binding protein 51 regulates nuclear transport of the glucocorticoid receptor beta and glucocorticoid responsiveness," Invest. Ophthalmol. Visual Sci. 49, 1037-1047 (2008).

44. J. H. Fingert et al., "Evaluation of the myocilin (MYOC) glaucoma gene in monkey and human steroid-induced ocular hypertension," Invest. Ophthalmol. Visual Sci. 42, 145-152 (2001).

45. A. R. Shepard et al., "Delayed secondary glucocorticoid responsiveness of MYOC in human trabecular meshwork cells," Invest. Ophthalmol. Visual Sci. 42, 3173-3181 (2001).

46. B. J. Fan et al., "Gene expression profiles of human trabecular meshwork cells induced by triamcinolone and dexamethasone," Invest. Ophthalmol. Visual Sci. 49, 1886-1897 (2008).

47. T. Ishibashi et al., "cDNA microarray analysis of gene expression changes induced by dexamethasone in cultured human trabecular meshwork cells," Invest. Ophthalmol. Visual Sci. 43, 3691-3697 (2002).

48. W. R. Lo et al., "Tissue differential microarray analysis of dexamethasone induction reveals potential mechanisms of steroid glaucoma," Invest. Ophthalmol. Visual Sci. 44, 473-485 (2003).

49. G. C. Munguba et al., "Nerve fiber layer thinning lags retinal ganglion cell density following crush axonopathy," Invest. Ophthalmol. Visual Sci. 55, 6505-6513 (2014).

50. G. Rovere et al., "Comparison of retinal nerve fiber layer thinning and retinal ganglion cell loss after optic nerve transection in adult albino rats," Invest. Ophthalmol. Visual Sci. 56, 4487-4498 (2015).

51. X.-R. Huang, R. W. Knighton, and L. N. Cavuoto, "Microtubule contribution to the reflectance of the retinal nerve fiber layer," Invest. Ophthalmol. Visual Sci. 47, 5363-5367 (2006).

52. X. R. Huang and R. W. Knighton, "Altered F-actin distribution in retinal nerve fiber layer of a rat model of glaucoma," Exp. Eye Res. 88, 11071114 (2009).

53. X. Huang et al., "Distortion of axonal cytoskeleton: an early sign of glaucomatous damage," Invest. Ophthalmol. Visual Sci. 52, 28792888 (2011).

54. J. Yi et al., "Optical detection of early damage in retinal ganglion cells in a mouse model of partial optic nerve crush injury," Invest. Ophthalmol. Visual Sci. 57(13), 5665-5671 (2016).

55. A. P. Cherecheanu et al., "Ocular perfusion pressure and ocular blood flow in glaucoma," Curr. Opinion Pharmacol. 13, 36-42 (2013).

56. B. E. Prum, Jr. et al., "Primary open-angle glaucoma preferred practice pattern ${ }^{\circledR}$ guidelines," Ophthalmology 123, P41-P111 (2016).

57. J. Flammer et al., "The impact of ocular blood flow in glaucoma," Prog. Retinal Eye Res. 21, 359-393 (2002).

58. G. Fuchsjäger-Mayrl et al., "Ocular blood flow and systemic blood pressure in patients with primary open-angle glaucoma and ocular hypertension," Invest. Ophthalmol. Visual Sci. 45, 834-839 (2004).

59. R. Haindl et al., "Total retinal blood flow in healthy and glaucomatous human eyes measured with three beam Doppler optical coherence tomography," in Biomed. Opt., Optical Society of America, Fort Lauderdale, Florida, TTh1B.2 (2016).

60. E. Vandewalle et al., "Oximetry in glaucoma: correlation of metabolic change with structural and functional damage," Acta Ophthalmol. 92, 105-110 (2014).

61. W. Liu et al., "Increased retinal oxygen metabolism precedes microvascular alterations in type 1 diabetic mice," Invest. Ophthalmol. Visual Sci. 58, 981-989 (2017).

Biographies of the authors are not available. 